\title{
A study on effectiveness of lactational amenorrhea as a method of contraception
}

\author{
Kamlesh Tiwari $^{1 *}$, Ishmat Khanam ${ }^{1}$, Neha Savarna ${ }^{2}$
}

\begin{abstract}
${ }^{1}$ Department of Obstetrics and Gynecology, Vardhman Institute of Medical Sciences, Pawapuri, Nalanda, Bihar, India ${ }^{2}$ Department of Preventive and Social Medicine, Darbhanga Medical College, Laheriasarai, Darbhanga, Bihar, India
\end{abstract}

Received: 24 August 2018

Accepted: 30 August 2018

\author{
*Correspondence: \\ Dr. Kamlesh Tiwari, \\ E-mail: tiwarikamlesh_2@rediffmail.com
}

Copyright: (c) the author(s), publisher and licensee Medip Academy. This is an open-access article distributed under the terms of the Creative Commons Attribution Non-Commercial License, which permits unrestricted non-commercial use, distribution, and reproduction in any medium, provided the original work is properly cited.

\begin{abstract}
Background: The lactational amenorrhea method, known as LAM, is part of the World Health Organization's list of accepted and effective methods of family planning. Studies show LAM to be $98 \%$ effective, as effective as the pill and other modern methods, when used according to guidelines. As LAM costs nothing, easy and natural to use, no medication or any devise needed, no interference with natural hormones, no interruption in conjugal life and more over the mother enjoying her motherhood life, it sounds very good as a method of contraception.

Methods: This is a prospective non-comparative study. A total of 298 mothers participated in this study. The benefits of exclusive breast feeding with particular emphasis on timely breastfeeding and must feeding during the night hours were explained to the mothers and the physiology of lactational amenorrhea was explained to them. All the mothers were interviewed and followed up on monthly basis.

Results: No lactating mothers conceived within the first six months of delivering their baby and only $4 \%$ mothers had evidence of pregnancy mainly after the 10th months after delivery.

Conclusions: The lactational amenorrhea method is a highly effective method of contraception for 6 months if exclusive breastfeeding practices are followed. In a country like India where use of contraceptive methods are very meager, LAM may prove a boon as it provides dual benefit of exclusive breastfeeding and prevention of pregnancy.
\end{abstract}

Keywords: Contraception, Exclusive breast feeding, Lactational amenorrhea method

\section{INTRODUCTION}

Lactation amenorrhea is a period which is literally defined as a period of suppression of menstruation in a woman due to lactation of her baby. This has been used as a temporary contraceptive method that relies on exclusive breast feeding usually from birth up to six months afterwards.

In 1988 in Bellagio (Italy) a conference was held to reach a consensus statement to define Lactational amenorrhea method as a method of contraception and it was stated that, a postpartum woman has at least 98 percent protection from pregnancy for six months when she remains amenorrheic and fully or nearly fully breastfeeds.

Further during that conference, the lactational amenorrhea method was defined as the informed use of breastfeeding as a contraceptive method by a woman who is still amenorrheic and does not feed her baby with supplements for up to six months after delivery.

The lactational amenorrhea method, known as LAM, is part of the World Health Organization's list of accepted and effective methods of family planning. Studies show LAM to be $98 \%$ effective, as effective as the pill and 
other modern methods, when used according to guidelines. $^{1-4}$

According to Labbok LAM is a transitional form of contraception and is most effective in women planning to breastfeed exclusively during the first six months. ${ }^{5}$

Producing milk is called lactation and not having a period is called amenorrhea, hence this method of birth control is called lactational amenorrhea.

It has been found that if a woman exclusively breastfeeds her baby for six months, the hormones that produce the breast milk also work to stop the ovaries from releasing ovum for the first six months after the birth of a baby and a woman doesn't has her period during that period.

The baby's age of 6 months is a somewhat an arbitrary choice, but it has been chosen because 6 months is the period during which the World Health Organization recommends mothers exclusively breastfeed for and after which time complementary foods must begin. However, studies have shown that, if women achieve 6 months of successful LAM use, continue their breastfeeding frequency when they introduce complementary feeding, and breastfeed first before the complementary food at each feeding, they may have continued high efficacy of the LAM.

LAM has been widely accepted as a natural family planning method that demands no abstinence. It is used as an introductory method for the postpartum period for the breastfeeding woman who hesitates to use a hormonal or chemical method. It has the added benefit of encouraging optimal breastfeeding behaviour, providing support for the health of the mother and the child.

The lactational amenorrhea method (LAM) is a highly efficient tool for the individual woman to utilize physiology to space births. Suckling induces a reduction in gonadotropin releasing hormone, luteinizing hormone and follicle stimulating hormone release, resulting in amenorrhea, through an intracerebral opioid pathway, beta-endorphins inhibit gonadotropin releasing hormone and dopamine secretions, which, in turn stimulates prolactin secretion and milk production. Reduced suckling precipitates the return of ovulation.

For lactational amenorrhea method to work, it has been suggested that that:

1. The baby must be under six months of age.

2. The mother must not have had a period.

3. The baby must be exclusively breastfed i.e. no added supplementation should be done, even during night.

Further it has been suggested that for best utilization of this opportunity as a method of contraception the mother must breast fed her baby at least every four hours during day time and at least every six hours during night.
When all these criteria are fulfilled LAM has been found to be effective in preventing pregnancy in the range of 98 to $99.5 \% .^{5}$

In women who do not experience menstrual bleeding and continue to breastfeed exclusively, lactational amenorrhoea provides contraceptive protection which is $94 \%$ effective, one year postpartum.

As LAM costs nothing, easy and natural to use, no medication or any devise needed, no interference with natural hormones, no interruption in conjugal life and more over the mother enjoying her motherhood life, it sounds very good as a method of contraception.

Many studies had been done on this method of contraception in various part of world but only few studies in our country and particularly our state, to conduct such type of studies is the need of hour.

The aim of the study is to determine the efficacy of the lactational amenorrhea method as a method of contraception in fully breastfeeding women.

\section{METHODS}

This is a prospective non-comparative study conducted in the department of obstetrics and gynaecology, Vardhman Institute of Medical Sciences, Pawapuri, Nalanda, Bihar, India during the period from January 2016 to March 2018.

During this period 368 mothers were enrolled for this study with informed consent. 12 mothers were excluded from study due to death of their babies, 36 mothers moved to such areas where regular follow up was not possible and 22 mothers later refused to participate in the study. Hence a total of 298 mothers participated in this study.

\section{Inclusion criteria}

- Willingness of the lactating mothers residing in the catchment area of VIMS, Pawapuri, who can be approached easily for follow up.

The benefits of exclusive breast feeding with particular emphasis on timely breastfeeding and must feeding during the night hours were explained to the mothers and the physiology of lactational amenorrhea was explained to them. Doubts regarding active sexual activities were fully explained and were encouraged to remain sexually active as per the wish of the couple. All the mothers were interviewed and followed up on monthly basis.

\section{RESULTS}

In this study $78.6 \%$ of mothers were below the age of 30 years $(54.4 \%$ in the age group of 18 to 25 years, and $24.2 \%$ in $25-30$ years). $19.5 \%$ mothers were in the age 
group of 30-35 years. Only $2 \%$ mothers were above 35 years. As this is a non-comparative observational study, there is no data to compare the observations in similar other mothers in the same area.

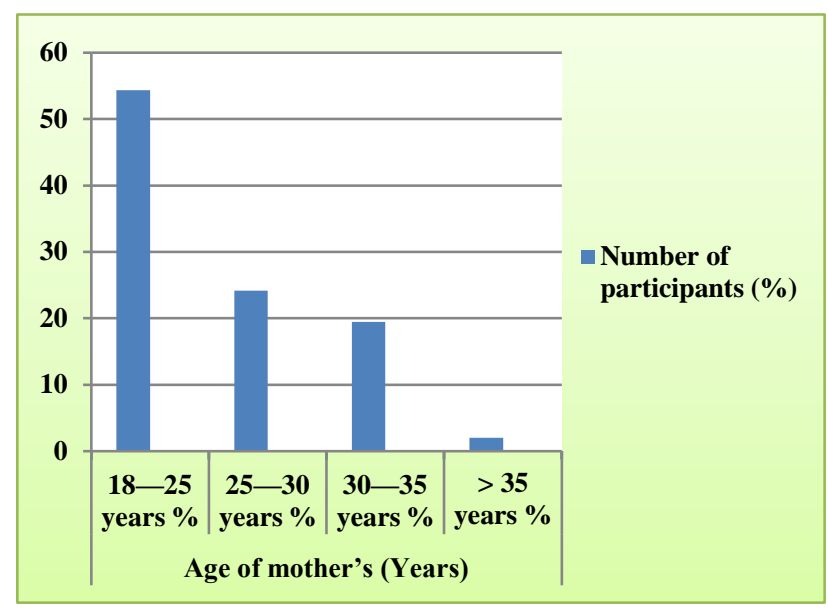

Figure 1: Age of mothers.

In this study $66 \%$ mothers were para- $1.21 \%$ mothers had parity -2 , while $13 \%$ mothers had parity more than 2 . As majority of mothers in this study $(74.6 \%)$ were in the age group of 18-30 years, hence $2 / 3$ rd mothers had parity of single viable child.

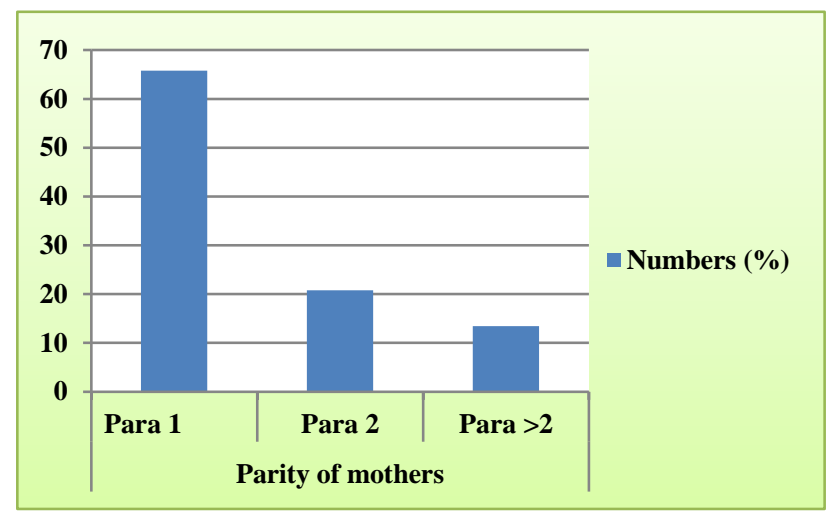

Figure 2: Parity of mothers.

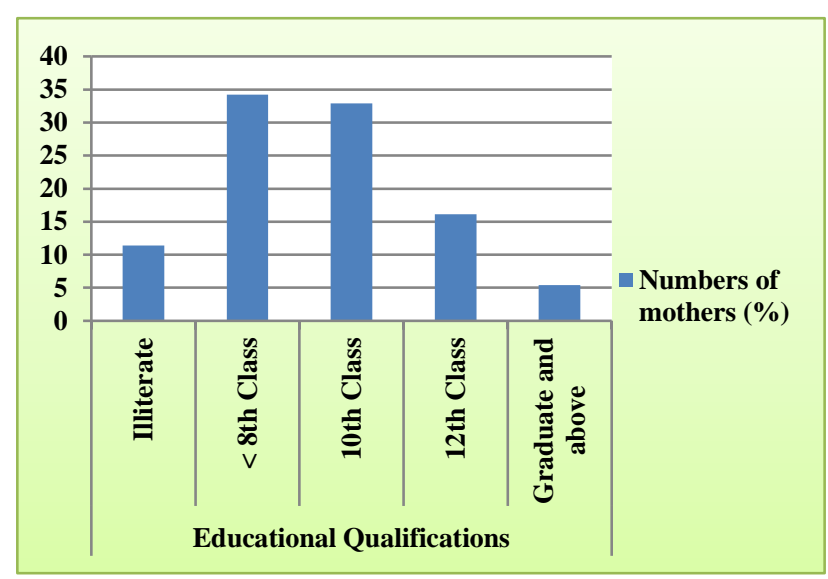

Figure 3: Educational qualifications.
Due to growing awareness and availability of Schools in rural areas about $67 \%$ mothers had education up to 10th standard or less, while $16 \%$ up to Class 12 . Only $5.4 \%$ mothers in this study had education up to graduation or above while $11.6 \%$ mothers were illiterate in this study.

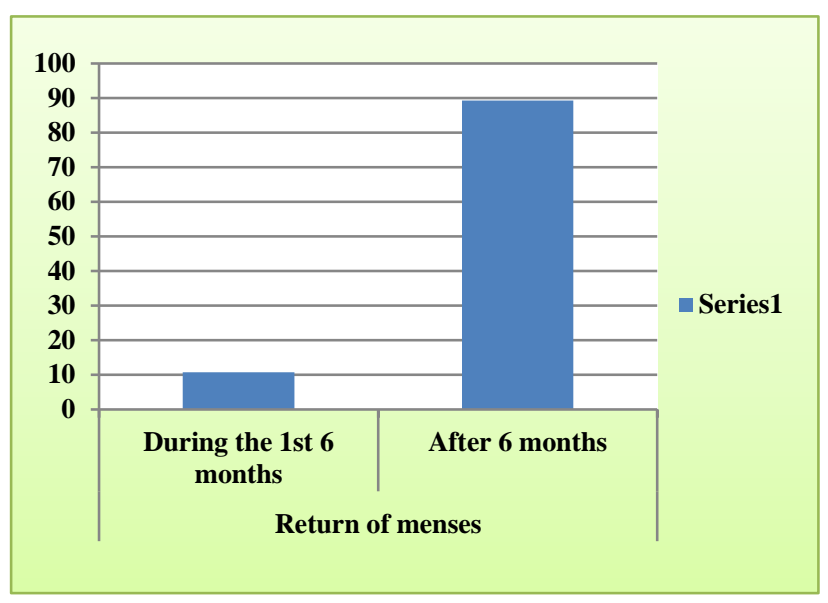

Figure 4: Return of menses.

In this study only $11 \%$ mothers had return of menstruation during the first six months of follow up. All but one of the mothers had return of menstruation after five months of exclusive breastfeeding, while in a solitary mother it was after 4 months. In $89 \%$ mothers the regular menstruation started after six months, in majority after 8 months of delivery. The mothers who were successful in starting complimentary feed more effectively as per counselling, the return of menses in them was earlier than who insisted more on their breastfeeding.

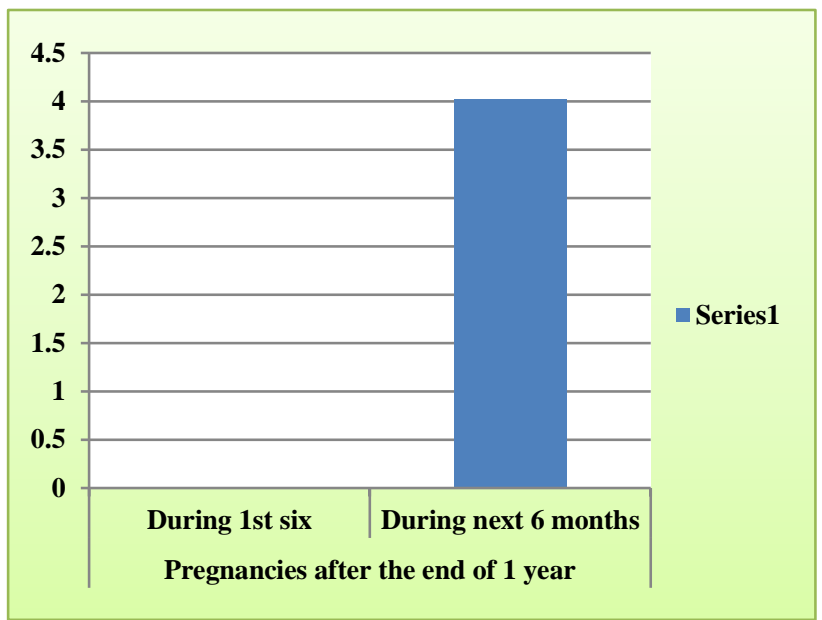

Figure 5: Pregnancies after the end of 1 year.

All the participating mothers were explained to live their sexual life freely as per their will without adopting any method of contraception other than using exclusive breastfeeding as per guidelines. In this study no lactating mothers conceived within the first six months of delivering their baby and only $4 \%$ mothers had evidence of pregnancy mainly after the 10 th months after delivery. 


\section{DISCUSSION}

Breast feeding is the one of the best gifts provided by the nature to mankind. Breast feeding not only provides the essential nutritional needs of the baby but also provides a very competent method of contraception during the initial period after child birth. As no medication is needed or any devise is used, if properly adhered to the basic principle of absolute breastfeeding, it is the best way of contraception.

In present study $54 \%$ of volunteers were in the age group of $18-25 \%$ and $24 \%$ in $25-30$ years age range, with $67 \%$ mothers having educational qualifications up to $10^{\text {th }}$ standard or less, $22 \%$ intermediate and above and only $11 \%$ unable to read and write. In a similar study in Pakistan the mean age of the volunteers was 27.2years and only $37.6 \%$ were able to read and write. ${ }^{6}$

In present study $65 \%$ mothers had parity 1 , while $21 \%$ were para 2. In the study from Pakistan the women reported an average of 4.4 pregnancies. $^{6}$

In the present study none of the mothers returned to menses during the first 6 months of postpartum. The study from Pakistan showed that $26.4 \%$ of the women returned to menses during the first 6 months postpartum. ${ }^{6}$

The $89 \%$ mothers in this study returned to menses after 6 months of postpartum with a total of $4.2 \%$ pregnancy. The study by Kazi A et al showed $7.2 \%$ pregnancies during that period. ${ }^{6}$

The level of education and socioeconomic status has direct effect on counselling with successful outcome on exclusive breastfeeding and late return of menses leading to low pregnancy rate in this study.

In present study none of the mothers had pregnancy during the exclusive breastfeeding period of first six months of postpartum with $4 \%$ pregnancies after 6 months during $1^{\text {st }}$ year of postpartum period. Kazi A et al observed that during full or nearly full breastfeeding, while the women were amenorrheic and not otherwise contracepting, the rate of pregnancy was $0.6 \%$. The pregnancy rate during lactational amenorrhea alone was $1.1 \%$ at 1 year postpartum. ${ }^{6}$

In the study by Vekemans $M$ the lactational amenorrhea method was found at least $98 \%$ effective, comparing favorably with other contraceptive methods. ${ }^{7}$ The author observed that lactational amenorrhea method gives time to decide upon a long-term method of contraception. Introduction of LAM in family planning programs demands training, attention to be given to working mothers, positive attitudes of health personnel, close links between postpartum and family planning teams, situation analysis, budgets, evaluations, follow-up activities, modifications of record keeping systems and computing programs, and of national family planning guidelines. In conclusion, LAM is an efficient family planning method which should be promoted. The lactational amenorrhea method should always include the shift to another method when its criteria are no longer implemented. ${ }^{7}$

Trussell $\mathbf{J}$ observed that the women who follow the suggestions and meet the criteria of exclusive breastfeeding, LAM is $>98 \%$ effective during the first six months postpartum. ${ }^{8}$

The $98+\%$ efficacy of LAM has been confirmed in a wide variety of settings. In addition, the results yield insight on the possibility of continued use beyond 6 months. LAM is found to be highly effective as an introductory postpartum method when offered in a variety of cultures, health care settings, socio-economic strata, and industrial and developing country locales. In addition, LAM acceptance complements breastfeeding behaviors without ongoing breastfeeding support services. ${ }^{5}$

Bracher et al, Kennedy et al have observed that implications for practice fully breastfeeding women who remain amenorrheic have a very small risk of becoming pregnant in the first six months after delivery when relying on lactational subfertility. ${ }^{9-11}$

This study, although only very small in size localized in a particular geographical area and covering mothers of almost similar socioeconomic profile, has a strong massage that if proper and sustained counselling is done the dual purpose of exclusive breastfeeding and a method of natural family planning can be achieved. With birth spacing the Infant and Young child feeding practices can be followed successfully and thus we can be able to achieve a better healthy future generation of the country.

\section{CONCLUSION}

The lactational amenorrhea method is a highly effective method of contraception for 6 months if exclusive breastfeeding practices are followed. Even after 6 months of exclusive breastfeeding period when complimentary feed is initiated, a high degree of contraceptive protection endures for a full year during lactational amenorrhea. In a country like India where use of contraceptive methods is very meagre, LAM may prove a boon as it provides dual benefit of exclusive breastfeeding and prevention of pregnancy.

\section{ACKNOWLEDGMENTS}

Authors would like to acknowledge the resident doctors and nursing staffs working in the Department of Obstetrics and Gyanecology, Vardhman Institute of Medical Sciences, Pawapuri, for their contribution in counselling the mothers and taking pain for proper follow up and special thanks to Mr. Rahul Kumar for his help in computer works.

\section{Funding: No funding sources}


Conflict of interest: None declared

Ethical approval: The study was approved by the Institutional Ethics Committee

\section{REFERENCES}

1. Kennedy KI, Rivera R, McNeilly AS. Consensus statement on the use of breastfeeding as a family planning method. Contracept. 1989;39:477-96.

2. Labbok M, Cooney K, Coly S. Guidelines: breastfeeding, family planning, and the Lactational Amenorrhea Method-LAM. Washington, DC: Institute for Reproductive Health; 1994.

3. American Academy of Pediatrics Section on Breastfeeding. Breastfeeding and the use of human milk [Policy statement]. Pediatr. 2012;129:e827-41.

4. Family Health International. Consensus statement: breastfeeding as a family planning method. Lancet. 1988;2:1204-5.

5. Labbok MH, Hight-Laukaran V, Peterson AE, FletcherV, von Hertzen H, Van Look PF. Multicenter study of the lactational amenorrhea method (LAM): I. efficacy, duration, and implications for clinical application. Contracept. 1997;55:327-36.

6. Kazi A, Kennedy KI, Visness CM, Khan T. Effectiveness of the lactational amenorrhea method in Pakistan. Fertil Steril. 1995;64(4):717-23.
7. Vekemans M. Postpartum contraception: the lactational amenorrhea method. Eur J Contracept Reprod Health Care. 1997;2(2):105-11.

8. Trussell J. Contraceptive failure in the United States. Contracept. 2011;83(5):397-404.

9. Bracher M. Breastfeeding, lactational infecundity, contraception and the spacing of births: implications of the Bellagio Consensus Statement. Health Transition Review. 1992;2:19-47.

10. Kennedy KI, Kotelchuck M, Visness CM, Kazi A, Ramos R. Users' understanding of the lactational amenorrhea method and the occurrence of pregnancy. J Human Lact. 1998;14(3):209-18.

11. Kennedy KI, Kotelchuck M. Policy considerations for the introduction and promotion of the lactational amenorrhea method: advantages and disadvantages of LAM. J Human Lact. 1998;14(3):191-203.

Cite this article as: Tiwari K, Khanam I, Savarna N. A study on effectiveness of lactational amenorrhea as a method of contraception. Int J Reprod Contracept Obstet Gynecol 2018;7:3946-50. 